This manuscript is published as R. van der Kant, J. Van Durme, F. Rousseau, and J. Schymkowitz, "SolubiS: Optimizing Protein Solubility by Minimal Point Mutations," in In Vitro Mutagenesis Protocols, vol. 1873, no. 10, New York, NY: Springer New York, 2018, pp. 317-333.

\title{
SolubiS: optimising protein solubility by minimal point mutations
}

Rob van der Kant1,2,3, Joost van Durme 1,2,3, Frederic Rousseau'1,2,3 and Joost Schymkowitz ${ }^{1,2,3}$

1. VIB Switch Laboratory, Herestraat 49, B-3000 Leuven, Belgium

2. Department of Cellular and Molecular Medicine, KU Leuven, Herestraat 49, PO 802, B-3000 Leuven, Belgium

3. VIB-KU Leuven Center for Brain and Disease Research, KU Leuven, Herestraat 49, PO 802, B3000 Leuven, Belgium

\section{i. Abstract}

Protein solubility is adapted to endogeneous protein abundance in the cell where protein folding is also assisted by multiple chaperones. During recombinant protein production, purification and storage proteins are frequently handled at concentrations that are several orders of magnitude above their physiological concentration, often resulting in protein aggregation. Here we describe SolubiS, a method allowing 1) detection of aggregation prone linear segments within a protein sequence and 2) identification of mutations that abolish the aggregation propensity of these segments without affecting the thermodynamic stability of the protein. Provided the availability of structural information this method is applicable to all globular proteins including antibodies, resulting both in increased in vitro protein solubility but also in better protein production yields.

\section{ii. Key words}




\section{SolubiS, protein aggregation, protein design}

\section{Introduction}

Protein aggregation remains a major factor limiting the production and application of many proteins, including monoclonal antibodies and enzymes. Protein aggregates are assemblies consisting of native and/or unfolded proteins [1] that have a negative effect on stability, safety, titers, immunogenicity, manufacturability and solubility of biologics in general [2-6].

Currently, it is widely accepted that aggregation is mediated by the presence of aggregation- prone regions (APRs), which assemble by intermolecular $\beta$ structured interactions, forming the core of the aggregate. Although these APRs are mostly hydrophobic in nature, they additionally require other key properties as a high $\beta$-sheet propensity and a low net charge. Methods of aggregation prediction are tailored to identify APRs in the primary sequence, which are extensively reviewed elsewhere [7-9]. These methods predict the theoretical aggregation potential of proteins in the unfolded state, termed the "intrinsic aggregation propensity". Most of these methods are based on the aggregation of short stretches of amino acids in vitro, outside of the context of the protein.

To nucleate aggregation, an APR is required to be solvent exposed to allow the formation of stable interactions with other like sequences. Yet, in most globular proteins, these APRs are buried inside the hydrophobic core of the folded structure, where they are protected from nucleating aggregation by the thermodynamic stability of the protein $[8,10]$. Significant exceptions are active sites and protein-protein interaction sites [11-14], where functional requirements appear to lead to the occurrence of APRs that can be detrimental when the protein is not engaged in functional interactions. For example, at formulation conditions of therapeutic proteins, high concentration and the absence of ligand or other interaction partners, promote protein self-interaction and the formation of aggregates in the stock solutions [15].

Besides structural stabilisation, a number of additional mechanisms can contribute to suppress aggregation [16]. One of these mechanisms is the presence 
of aggregation gatekeeper residues, which are generally charged residues or proline residues that decelerate the aggregation reaction [17-20]. In natural protein sequences, such gatekeeper residues are strongly enriched at the flanks of APRs. Moreover, molecular chaperones, such as Hsp70, bind to exposed APRs, therefore preventing intermolecular assembly of APRs to nucleate aggregation [21]. Finally, protein turnover rates [22] as well as protein expression levels [23] are evolutionary adapted to minimise protein aggregation under physiological conditions.

The SolubiS method allows for the introduction of gatekeeper residues in structurally unstable APRs, while maintaining the proteins structural integrity and function (Figure 1).

\section{The SolubiS theory in short}

Aggregation is a stochastic process, this means the effective aggregation of a protein is dependent on the population of aggregation-compatible conformations in which the APRs are exposed. The cooperation of physicochemical parameters such as protein and ion concentrations, $\mathrm{pH}$, and temperature contributes to determine the concentration of aggregation-prone conformers in a protein solution. Therefore, the challenge for relatively thermodynamically stable proteins is identifying sequences that will aggregate under native conditions. The solution to this challenge lies in the distinction between APRs that are thermodynamically protected by folding and those that occur in aggregationcompetent conformations that can form without major unfolding transitions (Figure 2A).

\section{Methods}

The SolubiS method uses TANGO to predict APRs from the primary peptide sequence [24] and the thermodynamic contribution of these APRs to the stability of the protein using FoldX [25]. FoldX performs best on crystal structures, but can also perform on a reliable homology model when the crystal structure has not been elucidated. The prediction of the effect of a mutation on the thermodynamic 
stability of a structure is also performed using FoldX.

\subsection{APR prediction from primary sequence}

While there are many predictors for intrinsic aggregation propensity published, we prefer to use TANGO [24], as it predicts APRs with well-defined sequence boundaries, that is, with a definitive segregation between the APR and the surrounding residues. Additionally, and especially important for the purpose of identifying critical APRs, TANGO accomplishes high specificity and thus predicts few false-positive APRs [7]. Moreover, no other predictors have been tested in combination with FoldX, meaning that the success rates are unknown. The SolubiS method currently only uses TANGO to predict APRs in the primary sequence and subsequently uses it to predict the effect of gatekeeper mutations on these APRs. The added benefit is a clear metric for the effect of each mutation, which makes it more straightforward to select a mutant that decreases the aggregation potential the most.

\subsection{Structural context}

Previous successful applications of the SolubiS methodology have been accomplished by starting with a high resolution crystal structure $[15,26]$. In the absence of a high-resolution structure, the best way to go is building a reliable homology model. Many methods have been developed to obtain such a homology model [25, 27-29], with the I-TASSER suite ranking number one in the most recent community-wide protein structure prediction experiment, CASP12. In the SolubiS method, as FoldX is used to predict the effect of a mutation on the thermodynamic stability of a protein, a model built or optimised by FoldX is preferred. In order to achieve optimal results, it is evident that a model should resemble the natural occurrence of the protein, meaning the oligomerisation state of the protein should be respected. To generate a reliable structure, attention should also be paid to crystal contacts, i.e. to artefactual protein interactions resulting from protein crystallisation, or by fusion proteins, stabilising nanobodies, molecular crowders and others. Conversely, attention should be paid to leave natural ligands or cofactors in place. Once a reliable and clean structure is obtained, the structure should be "repaired" using FoldX. FoldX uses a rotamer database, which contains 
a large set of side chain rotamers, extracted from high-resolution PDB structures. This process optimises the conformation of the side-chains, allowing for accurate calculation of the effect of each mutation. It is always wise to use different structures to perform the mutation scan and to compare the consistency of the predictions afterwards, thereby decreasing the risk of selecting destabilising mutations.

\subsection{Stretch-plots}

To easily visualise the aggregation susceptibility of a protein in function of both its intrinsic aggregation propensity and thermodynamic stability, we devised the "Stretch-plot" (Figure 2B). In a Stretch-plot, each APR is represented by a single data point, with on the $y$-axis the local "intrinsic" aggregation propensity of the sequence segment (summed TANGO, 5-100 per residue) and on the x-axis its contribution to the free energy of folding ( $\left.\Delta \mathrm{G}^{\text {contrib}}\right)$. The stretch-plot thereby allows for a simple description of the aggregation potential of each APR within a protein. As mentioned before, for a protein to aggregate, an APR has to be solvent exposed in order for it to interact with other like sequences. Local stability has been shown to be a good variable to predict the contribution of APRs to the aggregation propensity of a protein [15]. The stretch-plot therefore allows identifying and prioritising potential problematic APRs by both their intrinsic aggregation propensity and their contribution to the stability of the native protein. In theory, APRs in the top right corner are "critical" since they occur in less stable regions of the protein and display higher intrinsic aggregation propensities (Red dot in Figure 2B). A density plot of all stretch-plots based on a set of 2650 high resolution structures of globular proteins generated by the WhatIf software suite $[15,30]$, showed the distribution of APRs in globular proteins (Figure 2C). There is a clear tendency for proteins to have APRs located in the bottom left part of the stretch-plot, although there are many exceptions.

\subsection{Scoring function}

To extract critical APRs from the stretch-plot of each protein and represent these by a single metric, we developed a simple integrative scoring function. A corrected TANGO score for each APR was constructed by cutting off the $\Delta G^{\text {contrib }}$ at - 5 and + 
$5 \mathrm{kcal} / \mathrm{mol}$ and by normalising this value between 0 and 1 (0 representing a thermodynamically stable APR, 1 representing a highly unstable APR). Multiplying this value with the TANGO score allows the reduction of stable APRs in a way that the total score is dominated by the contribution of critical APRs. We call this scoring scheme the "SolubiS score". APRs with a low SolubiS score are deemed non-critical, i.e. having a good local stability. Low scoring APRs are usually buried in the core of the protein, where they contribute to stability. As they are unlikely to become solvent exposed under native conditions, they are unlikely to be the drivers of aggregation. High scoring APRs have a high TANGO score, i.e. aggregation propensity, and have an unfavorable $\Delta \mathrm{G}^{\text {contrib }}$, i.e. do not contribute to the stability of the protein. These APRs might become solvent exposed and are more likely to drive aggregation. Each APR is scored and the scores of all APRs in the protein are summed. A low score means that the protein contains no "critical" APRs, while a high score represents a protein that might aggregate under native conditions. The score can be used to compare proteins that are relatively similar, as previously applied for monoclonal antibodies [15], where the SolubiS score was used to classify antibodies based on aggregation propensity.

\subsection{Predicting SolubiS mutations}

Once a reliable structure has been chosen, all APRs in the primary sequence have been located and their structural context has been determined, the mutant prediction can start. In general every APR is scanned for possible aggregation reducing mutations. Per APR each residue is mutated to a gatekeeper residue (Arg, Lys, Glu, Asp or Pro) (Figure 1B). For all mutations the effect on aggregation propensity (total TANGO score) and stability (FoldX output) should be assessed (Figure 1C). To easily select mutations one can plot the $\Delta \Delta \mathrm{G}\left(\Delta \mathrm{G}_{\text {Mutant }}-\Delta \mathrm{G}_{\mathrm{WT}}\right)$ against the $\triangle$ TANGO $\left(\right.$ TANGO $_{\text {Mutant }}$ - TANGO ${ }_{\mathrm{WT}}$ ), resulting in a MASS-plot (mutant aggregation and stability spectrum) for each APR (Figure 2D) [15]. Three variables are important when considering which mutation to select, 1) the effect of each mutation on the aggregation propensity, 2) the effect of a mutation on the thermodynamic stability of the protein, 3 ) the effect of a mutation on the stability of the complex between the protein and its possible interaction partners. 


\subsection{Mutant selection}

As a MASS-plot is generally obtained for each APR, the MASS-plot of the most critical APRs should be carefully examined. The perfect SolubiS mutation maximally decreases the aggregation propensity ( $\triangle$ TANGO) and does not destabilise the structure. This means the best candidate mutations are in the bottom left corner of the plot (Figure 2D). Concerning $\Delta \Delta \mathrm{G}$, it would be optimal to find a stabilising mutation, but most importantly it should not destabilise the protein. As this is not always possible, we prefer trying the least destabilising mutation and in the subsequent round, try to find a mutation that counters the destabilising effect of the SolubiS mutation.

\subsection{Pipelines}

There are several ways to obtain SolubiS mutations. The first is using the webserver [31] (http://solubis.switchlab.org/), which produces the full output and guarantees a proper use of the relevant software. It makes a report, including the stretch-plot and MASS-plots, which makes the selection of mutations straightforward. The second is using the SolubiS plugin for YASARA [32, 33], which is more interactive and has been thoroughly documented, guiding the user through the process step-by-step. The last possibility is running TANGO and FoldX manually, which allows for more control over all steps. As it requires some scripting, output analysis and plotting, we suggest this method should only be used by people with substantial experience in protein structure bioinformatics. A benefit of this method is that one can use different aggregation propensity predictors, as well as different in silico predictors for calculating the effect of point mutations on the thermodynamic stability of a protein.

\subsection{Validating promising SolubiS mutations}

After the in silico analysis is performed and the data is collected, one can start choosing promising mutations. In our experience, selecting about five of the best ranking mutations generally results in the identification of a mutant with significantly decreased aggregation propensity. This number is only an indication to increase the success rate of the effort. Obviously these mutant proteins should 
be produced and purified, after which aggregation propensity, thermodynamic stability and functionality should be tested and compared to the wild type. In our experience, the largest jump in aggregation resistance is obtained when multiple SolubiS mutations are combined. As mentioned before, globular proteins have multiple aggregation prone regions, while one SolubiS mutation can only target one APR at the time. Generally we combine successful SolubiS mutations, resulting in a synergy effect. As multiple APRs can be available to cause aggregation, knocking one out might reduce the aggregation propensity, but the largest effect can be seen when removing both APRs. An additional factor to keep in mind is the effect of a SolubiS mutation on the isoelectric point (pI). Depending on your buffer $\mathrm{pH}$, a mutation can cause the $\mathrm{pI}$ to come closer to the $\mathrm{pH}$, thereby reducing the absolute net charge of the protein. This decreases the electrostatic repulsion between proteins, allowing them to get closer together, increasing the stochastic chance of interaction.

\section{Case Studies}

\section{1 $\alpha$-Galactosidase ( $\alpha$-Gal)}

Human $\alpha$-Galactosidase ( $\alpha$-Gal) is a lysosomal hydrolase whose deficiency results in Fabry disease, a metabolic X-linked inherited lysosomal storage disorder (LSD) $[34,35]$. Enzyme replacement therapy, where recombinantly produced $\alpha$-Gal is intravenously delivered to the patient to replace the endogenous protein, is currently used to treat Fabry disease. The structure of $\alpha$-Galactosidase consists of a homodimer in which each monomer contains a $(\beta / \alpha)$ domain (Figure 3A), harboring the active site and an antiparallel $\beta$ domain. Since the wild type protein was aggregation prone, we used the SolubiS method to design mutations to suppress this aggregation [26]. $\alpha$-Gal has three APRs, two of which are targetable by SolubiS. During the first round, single SolubiS mutations were selected, resulting in an increase in solubility, while retaining their enzymatic function. In the second round, double mutants and triple mutants were designed by combining the best mutations with additional mutations designed to increase the thermodynamic stability of the protein. As in the first round of mutations, the specific enzymatic activity remained comparable to WT. Interestingly the fraction of protein in the insoluble fraction was clearly reduced, and the accumulation of 
soluble aggregates in a size exclusion assay was clearly reduced as well.

\subsection{Protective Antigen (PA)}

PA is an element of the protein toxin secreted by Bacillus anthracis, the pathogen that causes anthrax [36]. PA is an important component of recombinant Anthrax vaccines, since the immune response against this virulence factor confers protection against Bacillus anthracis infection [37]. However, recombinant PA (rPA) stored in aqueous buffers is aggregation-prone [38]. The structure of PA reveals 4 domains (Figure 3B): Domain 1 contains two calcium binding sites and the furin cleavage site required for proteolytic activation of the molecule. Domain 2 is required for membrane insertion and together with domain 3 for heptamer formation. Domain 4 is essential for binding cell surface receptors [39]. TANGO detects several APRs in the amino acid sequence of the protein, of which one clearly stands out in the stretch plot (Figure 3B). One APR is located towards the top right, indicating low stability and high intrinsic aggregation propensity. Incidentally, previous experimental work identified this region as the key determinant of rPA aggregation in vitro [40]. Using SolubiS, three mutations were designed to reduce the aggregation propensity of PA, resulting in mutants that were more resistant to heat-stress-induced aggregation. An additional mutation was designed using FoldX to increase the thermodynamic stability of mutant PA, resulting in an improved thermodynamic stability and increased aggregation resistance at 40C. The scoring function facilitates converting the stretch-plot to a single value, the SolubiS score (Table 1). From both the stretch-plots and the SolubiS scores for WT and mutant PA, we can see the mutant is predicted to be significantly less aggregation prone than the wildtype. The "critical" APR is clearly visible in the WT stretch-plot, it has a high TANGO score and a low stability according to FoldX. This APR has disappeared in the stretch-plot of the aggregation resistant mutant, resulting in a much lower SolubiS score.

\section{$4.3 \beta 2$-Microglobulin $(\beta 2 \mathrm{~m})$}

Although the study by Radford and co-workers into the differences in aggregation between Human $\beta 2$-microglobulin $(\mathrm{h} \beta 2 \mathrm{~m})$ and Mouse $\beta 2$-microglobulin $(\mathrm{m} \beta 2 \mathrm{~m})$ 
was not designed with SolubiS in mind, it provides an interesting case for post-hoc analysis. $\mathrm{h} \beta 2 \mathrm{~m}$ is an aggregation-prone protein involved in pathological amyloid deposition in hemodialysis patients. $\mathrm{m} \beta 2 \mathrm{~m}$ on the other hand barely forms amyloid aggregates under physiological conditions despite having 100 times higher serum levels than $\mathrm{h} \beta 2 \mathrm{~m}$, which is more than five times higher than required to trigger dialysis-related $\mathrm{h} \beta 2 \mathrm{~m}$. Mouse and human $\beta 2$-microglobulin orthologs are $70 \%$ identical in sequence and share $88 \%$ overall sequence similarity. Considering only the primary structure, both sequences are predicted to have similar aggregation and amyloid propensities by various algorithms. Differences in aggregation propensity have been attributed to differences in solubility of the unfolded proteins at $\mathrm{pH} 2.0$ due to differences in (charge) composition of the FG-loop [41]. Analysing the structural context of each APR for the human (Figure 4A) and the mouse variant (Figure 4B) by means of stretch plots (Figure 4C and 4D, respectively), there also appears to be a large difference in the local contribution of the APR to the stability of the folded protein under physiological conditions: the APR in $h \beta 2 \mathrm{~m}$ is less stable than the APR in $\mathrm{m} \beta 2 \mathrm{~m}$, explaining the ability of $\mathrm{m} \beta 2 \mathrm{~m}$ to achieve much higher serum levels without forming amyloid deposits. In line with the experiments performed with these two proteins, the SolubiS score is high for $\mathrm{h} \beta 2 \mathrm{~m}$ and zero for $\mathrm{m} \beta 2 \mathrm{~m}$ (Table 1).

\subsection{Monoclonal antibodies}

Monoclonal antibodies are the largest class of protein therapeutics and continue to grow. Antibodies or immunoglobulins are large Y-shaped polypeptides composed of two heavy chains, containing four Ig-domains each, and two light chains, containing two Ig domains each (Figure 5A). This backbone structure is relatively constant and evolutionarily optimised to be soluble at the concentrations required in the blood. However, the antigen binding loops, or complementarity determining regions (CDRs) are hypervariable sequences that make up the loops of the terminal Ig domains of both heavy and light chains. We recently analysed experimentally characterised antibody sequences and showed that the aggregation behavior of monoclonals under mild temperatures is largely determined by the APRs that occur in the CDRs, since they are selected ad hoc to 
provide good binding to a specific antigen and are not optimised for solubility (Figure 5B). We demonstrated that the SolubiS score can be used to accurately distinguish between aggregation prone and not-aggregation prone monoclonal sequences, potentially providing a method to prioritise candidates for drug development [15]. Moreover, we analysed a monoclonal against the vascular endothelial growth factor VEGF in more detail. This antibody contained two APRs in the complementarity determining regions (CDRs) and stood out in the stretchplot (Figure 5B \& C). We used SolubiS to design mutations targeting both APRs (Figure 5D), which resulted in mutant antibodies with improved aggregation resistance under temperature stress as well as long-term storage conditions. However, perhaps equally important, the production yield of these mutants was up to five-fold higher than the wild type. Single mutants showed significant improvements in biophysical properties, but once more the combining of mutations resulted in the largest improvements.

\subsection{Antibody variable domains}

A final example can be found in mutational studies by Dudgeon et al aimed at lowering the aggregation of antibody variable domains by combinatorial means [2]. Phage display was used to find mutations that decreased the aggregation propensity of human antibody variable domains. Variable domains were chosen that belong to the most common families in human repertoire $\left(V_{H} 3, V_{\kappa} 1\right)$. Mutations that protected against aggregation clustered in the light chain variable domain CDR2 and in the heavy chain variable domain CDR1. Two mutations were found that improved the variable domain the most (52D in the light chain and 30D in the heavy chain). These findings were retrofitted to a single chain $F_{V}\left(\mathrm{scF}_{v}\right)$ of trastuzumab (Herceptin), a monoclonal that binds the extracellular domain of the HER2 receptor (Figure 6A). The mutations reduced the aggregation propensity of Herceptin and the stretch-plots of WT Herceptin (Figure 6B \& C) and its double mutant can confirm that, as well as the SolubiS Score (Table 1). Interestingly these two mutations have been patented (StAbilize ${ }^{\mathrm{TM}}$ Technology) and gave rise to a company (Solvanix). Coincidentally the APR in Herceptin CDR L2 is identical to the APR in mAb2 in [15], where different mutations were chosen to increase aggregation resistance and yield. 


\section{Conclusion}

Protein aggregation is a problematic phenomenon often observed when proteins are employed as therapeutic agents, thereby removing them from their native biological context and naturally occurring concentration. In other words, therapy often requires the production, storage and application of proteins above their natural solubility. And whereas buffer formulation can provide a quick fix to suppress the aggregation, this will not fundamentally eliminate the possibility. In contrast, altering the primary sequence has the potential to eliminate the aggregation propensity, increasing product safety.

SolubiS is a rational protein design method to minimise protein aggregation that combines prediction of aggregation hot spots in the primary sequence of a protein with the calculation of the thermodynamic stability of the structure. By screening many mutations in silico, we were able to show for diverse protein molecules, including monoclonal antibodies, that it is possible to significantly improve their aggregation behavior by a few well-chosen mutations, without affecting their desired function.

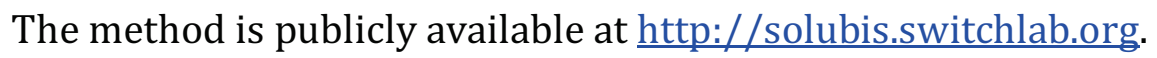

\section{Acknowledgements}

R.V.D.K. was supported by Boehringer Ingelheim Pharma GmbH \& Co. The VIB Switch Laboratory was supported by grants from VIB, University of Leuven, the Funds for Scientific Research Flanders (FWO), the Flanders Institute for Science and Technology (IWT) and the Federal Office for Scientific Affairs of Belgium (Belspo), IUAP P7/16, and by the European Research Council under the European Union's Horizon 2020 Framework Programme, ERC Grant agreement 647458 (MANGO) to J.S. 


\section{Figures}

Figure 1: The basic principle of the SolubiS method. A: Locate APRs using TANGO. B: Mutate every residue in an APR to the different gatekeeper residues (Arg, Lys, Asp, Glu and Pro). C: Calculate the effect of each mutation on the thermodynamic stability of the protein using FoldX.

Figure 2: Principles behind the SolubiS method. A: Example schematic representation of possible locations of APRs in proteins. APRs can occur buried in the protein (Blue), at the functional sites like domain interfaces (Orange) or at an unstable region of the protein (Red). B: Stretch-plots: representation of aggregation propensity and local stability of APRs. Problems increase toward the top right of the plot; ideally, APRs would be located in the bottom left. A single point in the stretch-plot represents an APR. In this theoretical representation the colors represent the APRs in Figure 2A. C: Density plot of aggregation propensity and local stability of APRs in crystal structure of globular proteins. The analysis is based on a set of 2650 high quality structures (R-factor of $<0.20$ and resolution of $<1.9$, with $30 \%$ sequence identity) of globular proteins generated by the Whatif software suite $[15,30]$. D: MASS-plots: representation for the potential aggregation reducing mutations in an APR. Green dots represent mutations that decrease the aggregation propensity, while not destabilising the structure. Red dots are mutations that either decrease the aggregation propensity insufficiently or are too destabilising to the structure.

Figure 3: Application of the SolubiS method on $\alpha$-galactosidase ( $\alpha$-Gal) and protective antigen (PA). A: Crystal structure of $\alpha$-Gal homodimer (PDBid: 3GXP [42]) with APRs highlighted. The small red circle shows the location of the two "critical" APRs, the middle red circle shows the same region zoomed in with the mutation sites highlighted. The respective APRs are highlighted in both the structure and the stretch-plot. The large red circle shows the same area in the aggregation resistant mutant (A348R/A368R/S405L). Mutations are highlighted in green. B: Crystal structure of PA wildtype (PDBid: 1T6B [43]). The small red circle shows the location of the "critical" APR in the stretch-plot (red dot). The large red circle shows the same area of the aggregation resistant mutant 
(T576E/S559L), based on a crystal structure (PDBid: 5FR3 [26]). Mutations are highlighted. Images of protein structures were generated using YASARA Structure [27].

Figure 4: Structural context of the APRs in Human and mouse $\beta 2$ microglobulin. A: Solution NMR structure ensemble of human $\beta 2$-microglobulin (PDBid: 2XKS [44]) showing the APR in red. B: Crystal structure of mouse $\beta 2$ microglobulin (PDBid: 1LK2 [45]) showing the APR in red. C: Stretch-plot of human $\beta 2$-microglobulin. D: Stretch-plot of mouse $\beta 2$-microglobulin. Images of protein structures were generated using YASARA Structure [27].

Figure 5: Application of the Solubis method on mAb2. A: Crystal structure of a full monoclonal antibody (PDBid: 5DK3 [46]). Blue: light chain, green: heavy chain. B: Crystal structure of the Fab region of mAb2 (PDBid: 2FJF [47]) with APRs highlighted. Small red circle shows the location of the "critical" APRs in the variable domain. C: Stretch-plot of the Fab region of mAb2 with the light chain critical APR indicated. D: Top view of the antigen-binding domain of a aggregation resistant mutant (SL50K/FH101P) with introduced mutations in green. Images of protein structures were generated using YASARA Structure [27].

Figure 6: Structural context of trastuzumab (Herceptin) APRs. A: Crystal structure of Herceptin wildtype Fab bound to its target. (PDBid: 1N8Z [48]). Orange: extracellular domain of human HER2 receptor, green: light chain, blue: heavy chain. B: Stretch-plot of Herceptin wildtype $\mathrm{F}_{\mathrm{V}}$ region based on the variable domains of a Herceptin crystal structure (PDBid: 4HKZ [49]). C: Location of "critical" APR in Herceptin wildtype $F_{V}$ in the light chain. Images of protein structures were generated using YASARA Structure [27]. 


\section{Tables}

\begin{tabular}{|c|c|c|}
\hline Name & PDBid & SolubiS score \\
\hline$\alpha$-Gal WT & $3 G X P[42]$ & 166.8 \\
\hline$\alpha-G a l$ A348R/A368R/S405L & FoldX [25] & 0 \\
\hline$\alpha-G a l$ A348R/A368P/S405L & FoldX [25] & 0 \\
\hline Protective Antigen WT & 1T6B [43] & 181.2 \\
\hline Protective Antigen T576E/S559L & 5FR3 [26] & 21.7 \\
\hline Human $\beta 2$-microglobulin & 2XKS [44] & 531.5 \\
\hline Mouse $\beta 2$-microglobulin & $1 \mathrm{LK} 2[45]$ & 0 \\
\hline mAb2 WT & $2 \mathrm{FJF}[47]$ & 277.0 \\
\hline mAb2 SL50K/FH101P & FoldX [25] & 30 \\
\hline Herceptin $\mathrm{F}_{\mathrm{V}} \mathrm{WT}$ & 4HKZ [49] & 234.0 \\
\hline Herceptin Fv SL52D/KH30D & $4 \mathrm{X} 4 \mathrm{Y}[50]$ & 78.8 \\
\hline
\end{tabular}

Table 1.1: SolubiS scores based on the stretch-plots of Figure 3-6. Names, representative structures, references and SolubiS scores are listed. For mutants where no structure has been elucidated, the mutation was done by FoldX on the representative wildtype structure and the SolubiS score was calculated based on the resulting mutant structure. 


\section{References}

1. Garidel P, Kebbel F (2010) Protein therapeutics and aggregates characterized by photon correlation spectroscopy. BioProcess Int

2. Dudgeon K, Rouet R, Kokmeijer I, et al (2012) General strategy for the generation of human antibody variable domains with increased aggregation resistance. Proc Natl Acad Sci USA 109:10879-10884. doi: 10.1073/pnas.1202866109

3. Kumar S, Singh SK, Wang X, et al (2011) Coupling of aggregation and immunogenicity in biotherapeutics: T- and B-cell immune epitopes may contain aggregation-prone regions. Pharm Res 28:949-961. doi: 10.1007/s11095-0110414-9

4. Vázquez-Rey M, Lang DA (2011) Aggregates in monoclonal antibody manufacturing processes. Biotechnol Bioeng 108:1494-1508. doi: 10.1002/bit.23155

5. Roberts CJ (2014) Protein aggregation and its impact on product quality. Curr Opin Biotechnol 30C:211-217. doi: 10.1016/j.copbio.2014.08.001

6. Wang W, Singh SK, Li N, et al (2012) Immunogenicity of protein aggregates-concerns and realities. Int J Pharm 431:1-11. doi: 10.1016/j.ijpharm.2012.04.040

7. Hamodrakas SJ (2011) Protein aggregation and amyloid fibril formation prediction software from primary sequence: towards controlling the formation of bacterial inclusion bodies. FEBS J 278:2428-2435. doi: 10.1111/j.17424658.2011.08164.x

8. De Baets G, Schymkowitz J, Rousseau F (2014) Predicting aggregation-prone sequences in proteins. Essays Biochem 56:41-52. doi: 10.1042/bse0560041

9. Belli M, Ramazzotti M, Chiti F (2011) Prediction of amyloid aggregation in vivo. EMBO Rep 12:657-663. doi: 10.1038/embor.2011.116

10. Fitzpatrick AW, Knowles TPJ, Waudby CA, et al (2011) Inversion of the Balance between Hydrophobic and Hydrogen Bonding Interactions in Protein Folding and Aggregation. PLoS Comput Biol. doi: 10.1371/journal.pcbi.1002169

11. Castillo V, Ventura S (2009) Amyloidogenic Regions and Interaction Surfaces Overlap in Globular Proteins Related to Conformational Diseases. PLoS Comput Biol. doi: 10.1371/journal.pcbi.1000476

12. Rousseau F, Wilkinson H, Villanueva J, et al (2006) Domain swapping in p13suc1 results in formation of native-like, cytotoxic aggregates. J Mol Biol 363:496-505. doi: 10.1016/j.jmb.2006.07.061

13. Wang X, Singh SK, Kumar S (2010) Potential Aggregation-Prone Regions in Complementarity-Determining Regions of Antibodies and Their Contribution Towards Antigen Recognition: A Computational Analysis. Pharm Res 27:15121529. doi: 10.1007/s11095-010-0143-5

14. Bennett MJ, Sawaya MR, Eisenberg D (2006) Deposition diseases and 3D domain swapping. Structure 14:811-824. doi: 10.1016/j.str.2006.03.011 
15. van der Kant R, Karow-Zwick AR, Van Durme J, et al (2017) Prediction and Reduction of the Aggregation of Monoclonal Antibodies. J Mol Biol 429:12441261. doi: 10.1016/j.jmb.2017.03.014

16. Balch WE, Morimoto RI, Dillin A, Kelly JW (2008) Adapting proteostasis for disease intervention. Science 319:916-919. doi: 10.1126/science.1141448

17. Otzen DE, Kristensen O, Oliveberg M (2000) Designed protein tetramer zipped together with a hydrophobic Alzheimer homology: a structural clue to amyloid assembly. Proc Natl Acad Sci USA 97:9907-9912. doi: 10.1073/pnas.160086297

18. Richardson JS, Richardson DC (2002) Natural beta-sheet proteins use negative design to avoid edge-to-edge aggregation. Proc Natl Acad Sci USA 99:2754-2759. doi: $10.1073 /$ pnas.052706099

19. Rousseau F, Schymkowitz J, Serrano L (2006) Protein aggregation and amyloidosis: confusion of the kinds? Current Opinion in Structural Biology 16:118-126. doi: 10.1016/j.sbi.2006.01.011

20. Monsellier E, Chiti F (2007) Prevention of amyloid-like aggregation as a driving force of protein evolution. EMBO Rep 8:737-742. doi: 10.1038/sj.embor.7401034

21. Van Durme J, Maurer-Stroh S, Gallardo R, et al (2009) Accurate prediction of DnaK-peptide binding via homology modelling and experimental data. PLoS Comput Biol 5:e1000475. doi: 10.1371/journal.pcbi.1000475

22. De Baets G, Reumers J, Delgado Blanco J, et al (2011) An evolutionary trade-off between protein turnover rate and protein aggregation favors a higher aggregation propensity in fast degrading proteins. PLoS Comput Biol 7:e1002090. doi: 10.1371/journal.pcbi.1002090

23. Tartaglia GG, Pechmann S, Dobson CM, Vendruscolo M (2009) A relationship between mRNA expression levels and protein solubility in E. coli. J Mol Biol 388:381-389. doi: 10.1016/j.jmb.2009.03.002

24. Fernandez-Escamilla A-M, Rousseau F, Schymkowitz J, Serrano L (2004) Prediction of sequence-dependent and mutational effects on the aggregation of peptides and proteins. Nat Biotechnol 22:1302-1306. doi: 10.1038/nbt1012

25. Schymkowitz J, Borg J, Stricher F, et al (2005) The FoldX web server: an online force field. Nucleic Acids Res 33:W382-8. doi: 10.1093/nar/gki387

26. Ganesan A, Siekierska A, Beerten J, et al (2016) Structural hot spots for the solubility of globular proteins. Nat Commun 7:10816. doi: 10.1038/ncomms10816

27. Krieger E, Vriend G (2002) Models@Home: distributed computing in bioinformatics using a screensaver based approach. Bioinformatics 18:315-318.

28. Arnold K, Bordoli L, Kopp J, Schwede T (2006) The SWISS-MODEL workspace: a web-based environment for protein structure homology modelling. Bioinformatics 22:195-201. doi: 10.1093/bioinformatics/bti770

29. Yang J, Yan R, Roy A, et al (2015) The I-TASSER Suite: protein structure and function prediction. Nat Methods 12:7-8. doi: 10.1038/nmeth.3213 
30. Hooft R, Sander C, Vriend G (1996) Verification of protein structures: Side-chain planarity. Journal of Applied Crystallography 29:714-716.

31. Van Durme J, De Baets G, van der Kant R, et al (2016) Solubis: a webserver to reduce protein aggregation through mutation. Protein Engineering Design and Selection 29:285-289. doi: 10.1093/protein/gzw019

32. De Baets G, Van Durme J, van der Kant R, et al (2015) Solubis: optimize your protein. Bioinformatics 31:2580-2582. doi: 10.1093/bioinformatics/btv162

33. Van Durme J, Delgado J, Stricher F, et al (2011) A graphical interface for the FoldX forcefield. Bioinformatics 27:1711-1712. doi: 10.1093/bioinformatics/btr254

34. Brady RO, Gal AE, Bradley RM, et al (1967) Enzymatic defect in Fabry's disease. Ceramidetrihexosidase deficiency. N Engl J Med 276:1163-1167. doi: 10.1056/NEJM196705252762101

35. Eng CM, Desnick RJ (1994) Molecular basis of Fabry disease: mutations and polymorphisms in the human alpha-galactosidase A gene. Hum Mutat 3:103-111. doi: 10.1002/humu.1380030204

36. Collier RJ (2009) Membrane translocation by anthrax toxin. Mol Aspects Med 30:413-422. doi: 10.1016/j.mam.2009.06.003

37. Brey RN (2005) Molecular basis for improved anthrax vaccines. Adv Drug Deliv Rev 57:1266-1292. doi: 10.1016/j.addr.2005.01.028

38. Singh S, Singh A, Aziz MA, et al (2004) Thermal inactivation of protective antigen of Bacillus anthracis and its prevention by polyol osmolytes. Biochem Biophys Res Commun 322:1029-1037. doi: 10.1016/j.bbrc.2004.08.020

39. Petosa C, Collier RJ, Klimpel KR, et al (1997) Crystal structure of the anthrax toxin protective antigen. Nature 385:833-838. doi: 10.1038/385833a0

40. Ganesan A, Watkinson A, Moore BD (2012) Biophysical characterisation of thermal-induced precipitates of recombinant anthrax protective antigen: evidence for kinetically trapped unfolding domains in solid-state. Eur J Pharm Biopharm 82:475-484. doi: 10.1016/j.ejpb.2012.05.019

41. Pashley CL, Hewitt EW, Radford SE (2016) Comparison of the aggregation of homologous $\beta 2$-microglobulin variants reveals protein solubility as a key determinant of amyloid formation. J Mol Biol 428:631-643. doi: 10.1016/j.jmb.2016.01.009

42. Lieberman RL, D'aquino JA, Ringe D, Petsko GA (2009) Effects of $\mathrm{pH}$ and iminosugar pharmacological chaperones on lysosomal glycosidase structure and stability. Biochemistry 48:4816-4827. doi: 10.1021/bi9002265

43. Santelli E, Bankston LA, Leppla SH, Liddington RC (2004) Crystal structure of a complex between anthrax toxin and its host cell receptor. Nature 430:905-908. doi: $10.1038 /$ nature 02763

44. Eichner T, Kalverda AP, Thompson GS, et al (2011) Conformational conversion during amyloid formation at atomic resolution. Molecular Cell 41:161-172. doi: 10.1016/j.molcel.2010.11.028 
45. Rudolph MG, Shen LQ, Lamontagne SA, et al (2004) A peptide that antagonizes TCR-mediated reactions with both syngeneic and allogeneic agonists: functional and structural aspects. J Immunol 172:2994-3002.

46. Scapin G, Yang X, Prosise WW, et al (2015) Structure of full-length human antiPD1 therapeutic IgG4 antibody pembrolizumab. Nat Struct Mol Biol 22:953-958. doi: $10.1038 /$ nsmb.3129

47. Fuh G, Wu P, Liang W-C, et al (2006) Structure-function studies of two synthetic anti-vascular endothelial growth factor Fabs and comparison with the Avastin Fab. J Biol Chem 281:6625-6631. doi: 10.1074/jbc.M507783200

48. Cho H-S, Mason K, Ramyar KX, et al (2003) Structure of the extracellular region of HER2 alone and in complex with the Herceptin Fab. Nature 421:756-760. doi: $10.1038 /$ nature 01392

49. Donaldson JM, Zer C, Avery KN, et al (2013) Identification and grafting of a unique peptide-binding site in the Fab framework of monoclonal antibodies. Proc Natl Acad Sci USA 110:17456-17461. doi: 10.1073/pnas.1307309110

50. Langley DB, Roome B, Christ D (2014) Retrofitting antibodies with stabilizing mutations. Herceptin scFv mutant K30D/S52D. RCSB PDB, To be published. doi: $10.2210 / \mathrm{pdb} 4 \mathrm{x} 4 \mathrm{y} / \mathrm{pdb}$ 
Van der Kant, Figure 1

A MYGSKAYVKHPADIPDYLKLSFPEGFKWE RVMNFEDGGVVTVTQDSSLQDGEFIYKVK LRGTNFPSDGPVMQKKTMGWEASSERMYP

B

$$
\text { VVTVT } \begin{cases}\text { PVTVT } & \text { KVTVT } \\ \text { VPTVT } & \text { VKTVT } \\ \text { VVPVT } & \text { VVKVT } \\ \text { VVTPT } & \text { VVTKT } \\ \text { VVTVP } & \text { VVTVK }\end{cases}
$$

C

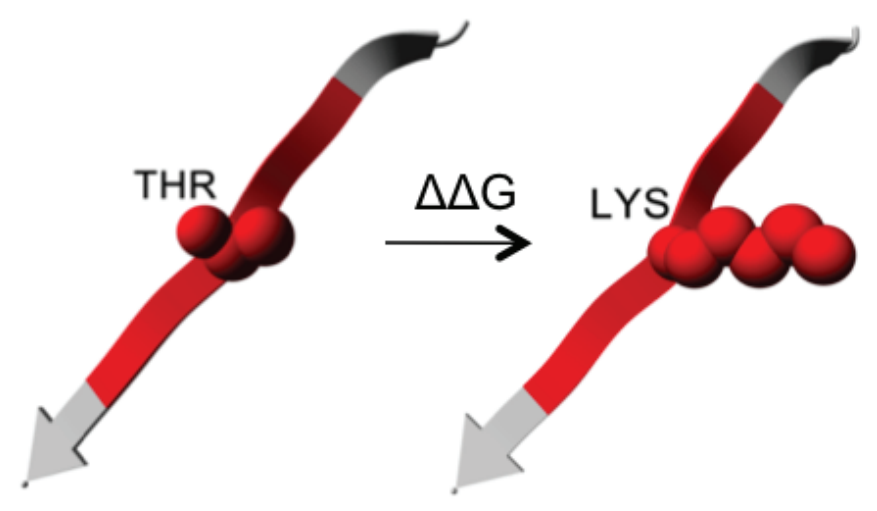


A
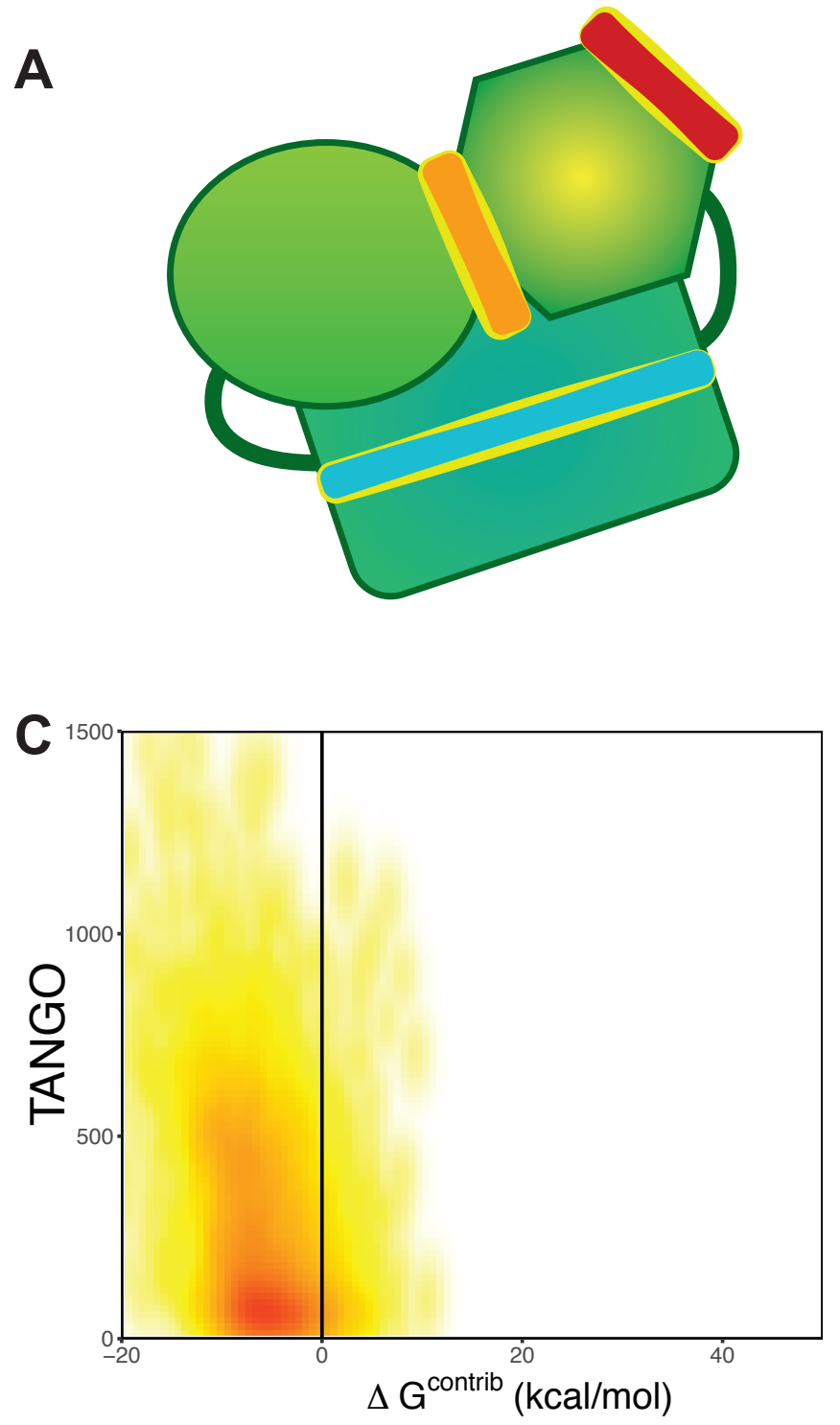

B

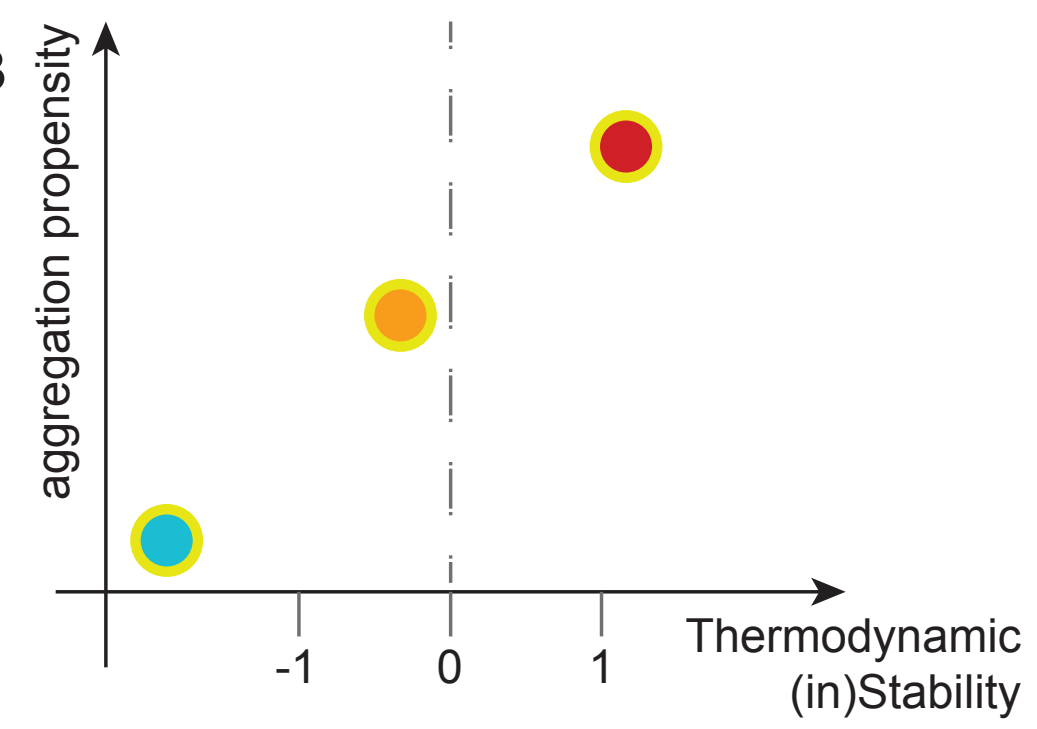

D

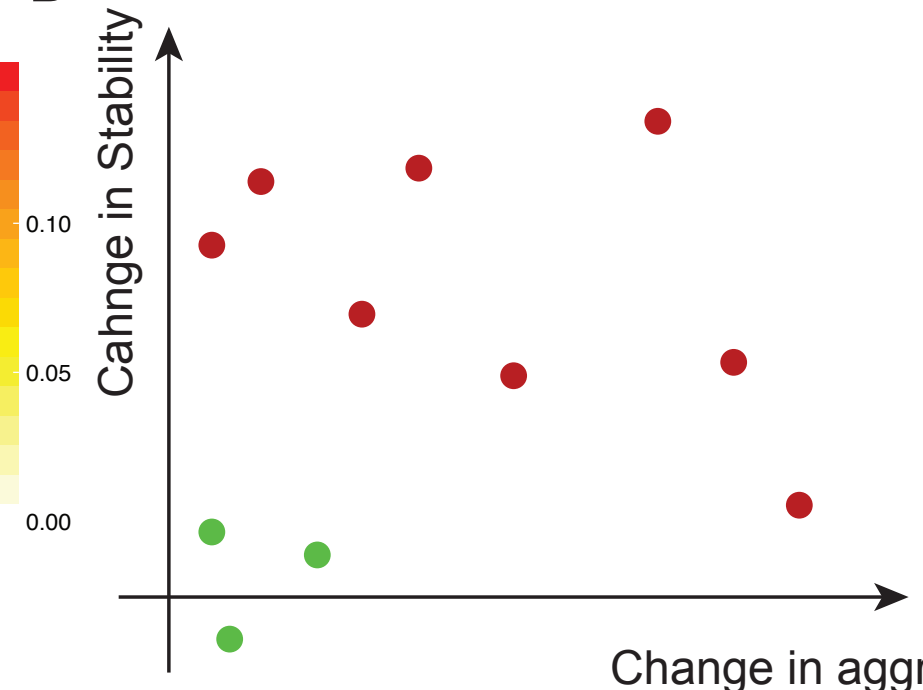
propensity 


\section{Van der Kant, Figure 3}

A
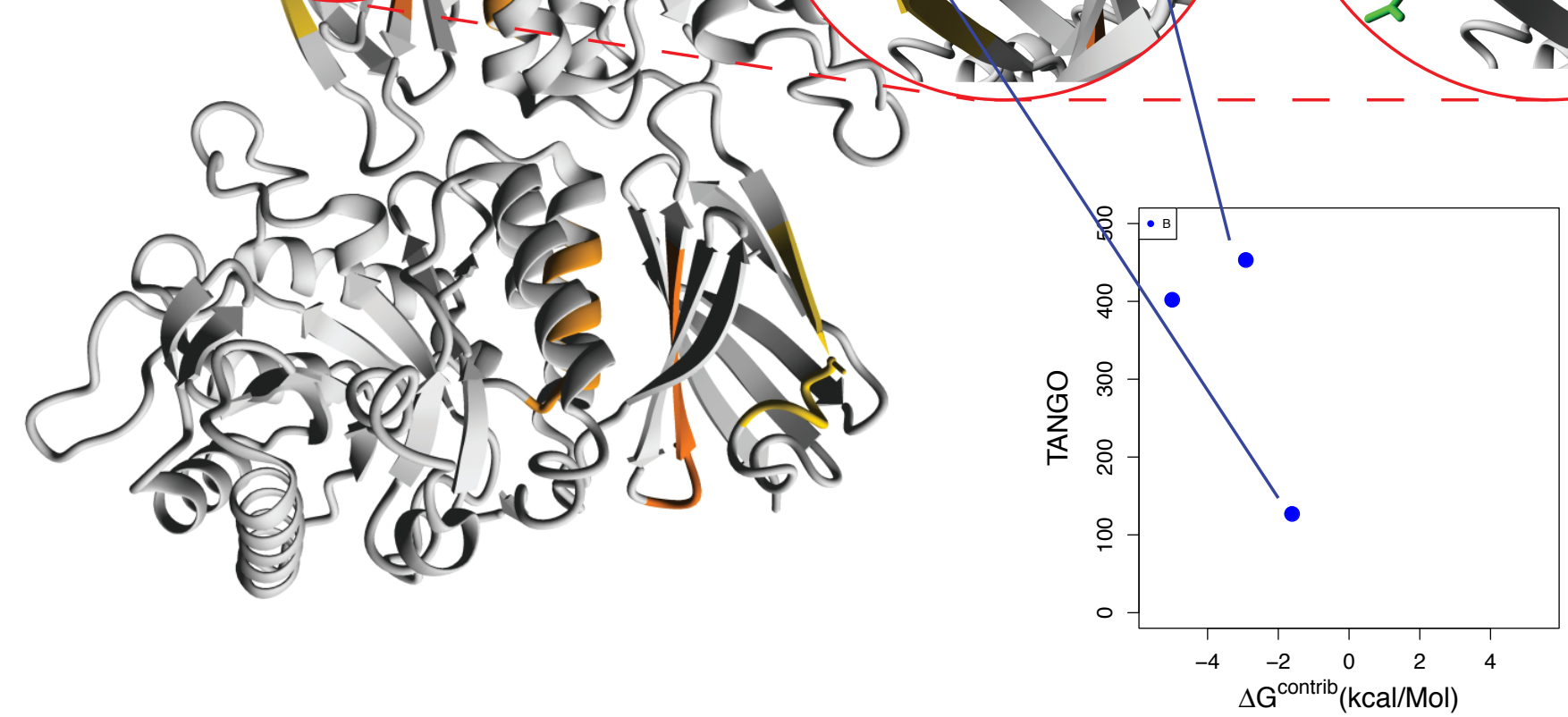

B

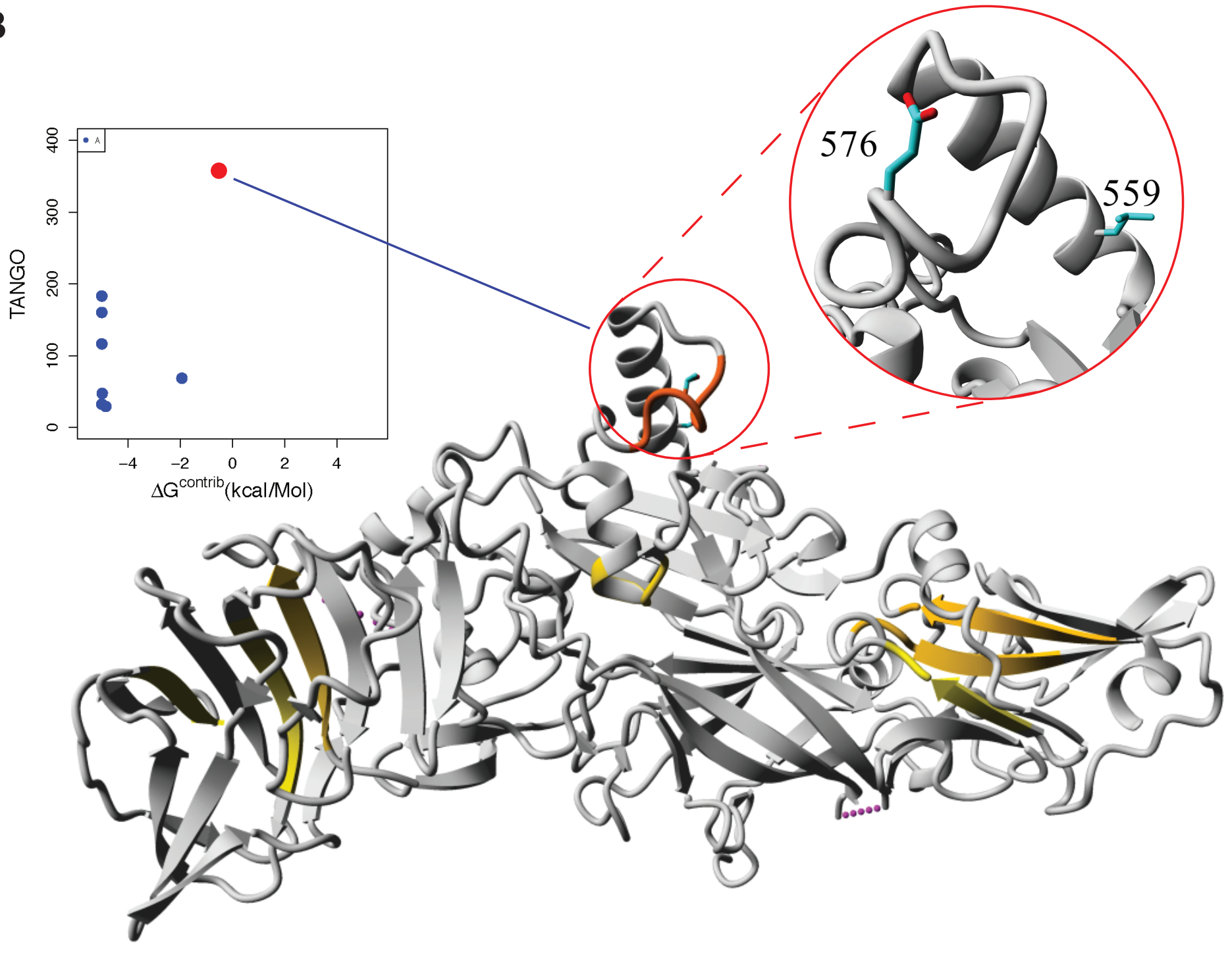


Van der Kant, Figure 4

A

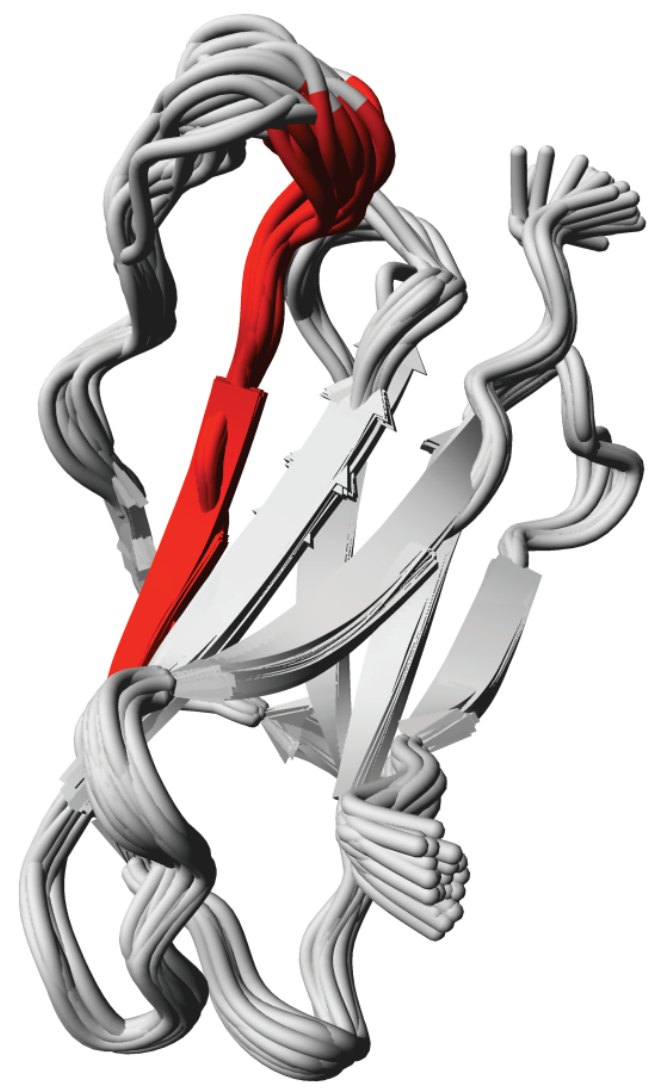

human
B

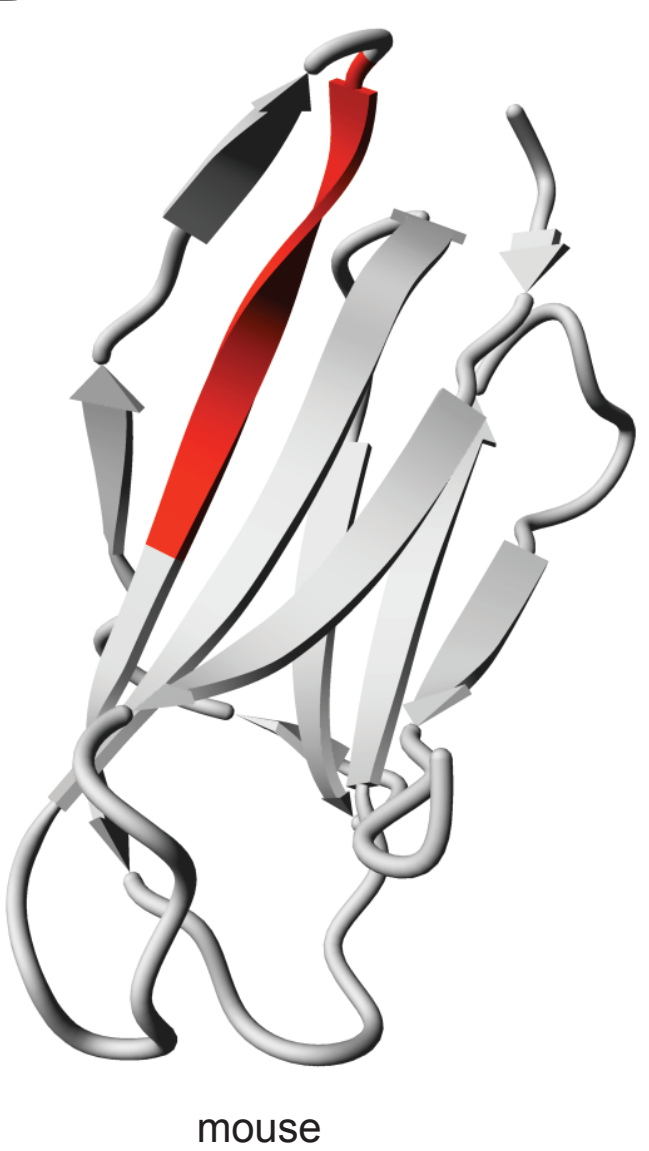

C
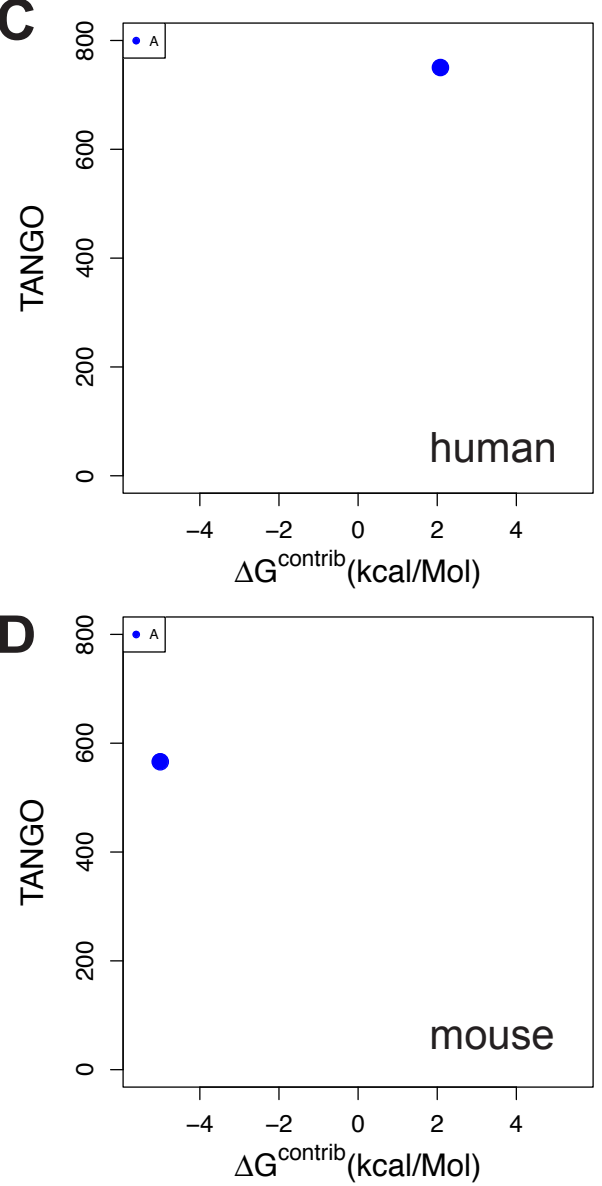


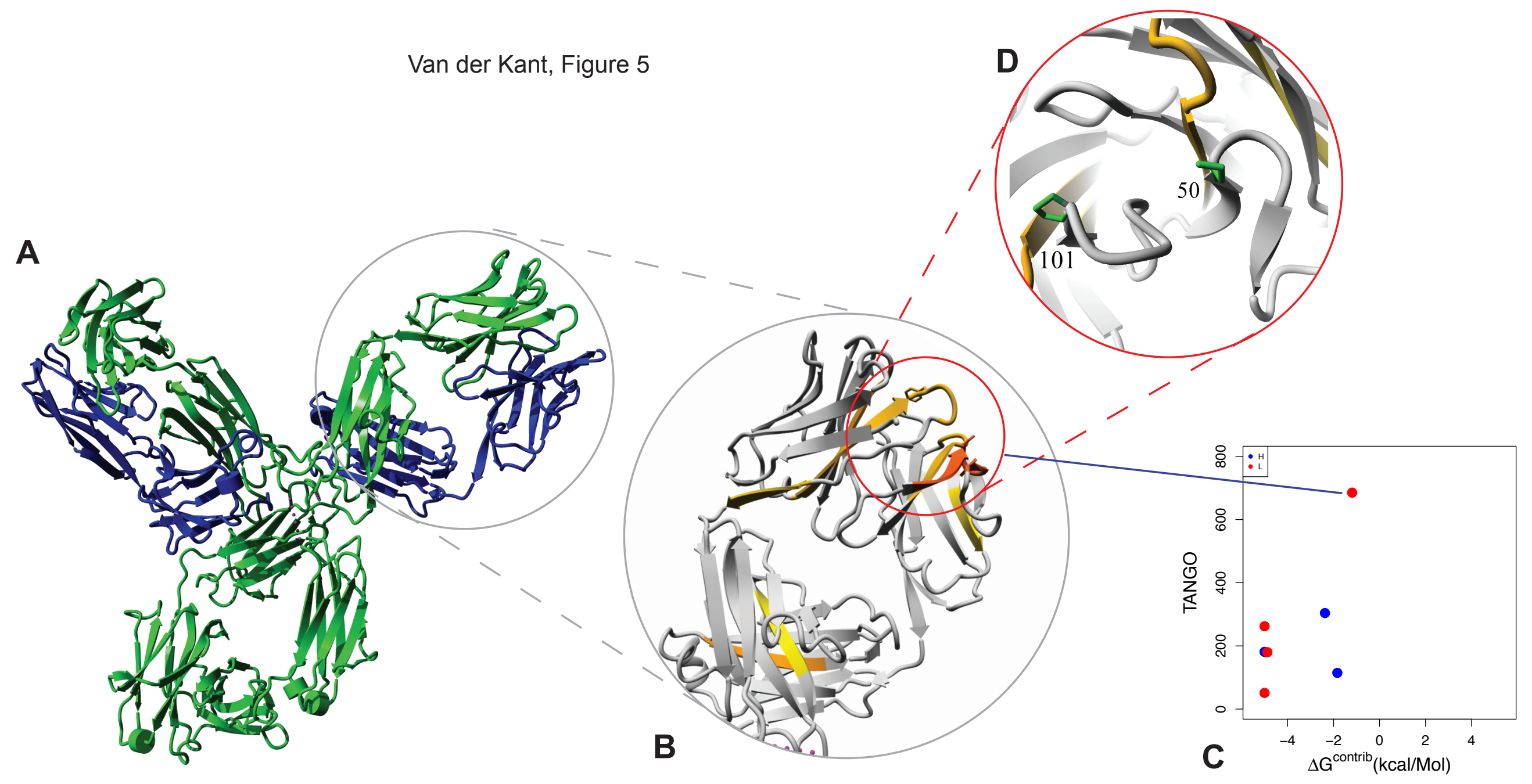




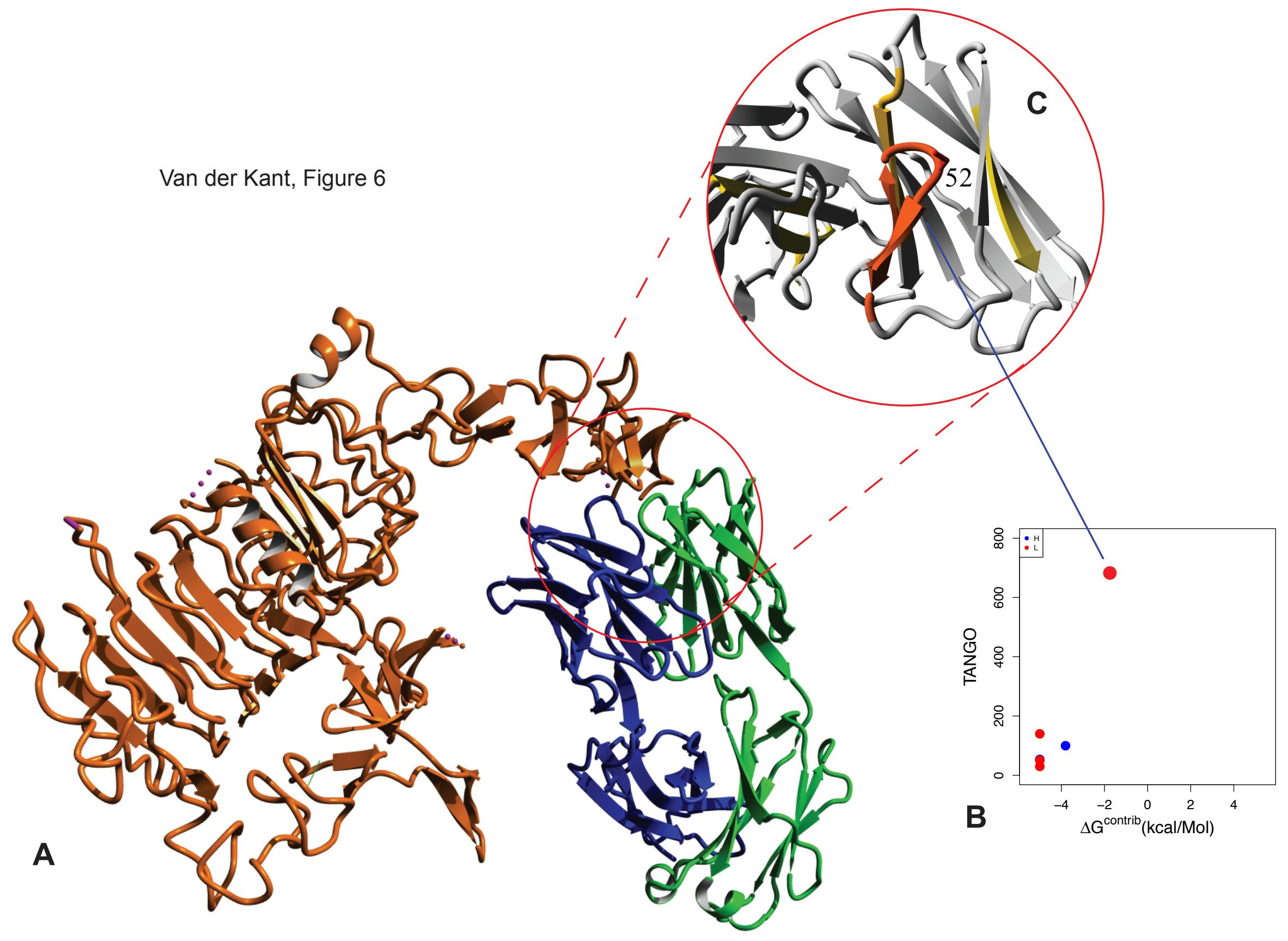

